\section{Geographic coding of addresses: A computer system}

REBECCA F. GUY and LOUIS G. POL Memphis State University, Memphis, Tennessee 38152

and

\section{CONRAD W. BRANDON \\ Memphis Methods, Memphis, Tennessee 38117}

Geographic coding of information has become an important tool in the analysis of data for federal, state, and local governments. Presently, there exist many individual street address records with valuable sociodemographic information, and frequently, analysts and planners desire to see these data aggregated by some type of areal unit (e.g., census tract, block, congressional district). Such aggregation permits a search for patterns with respect to the desired characteristics at various levels. Given the volume of records that often exists, it is imperative that a computer be available to perform this task. The U.S. Bureau of the Census (1970) has developed the ADMATCH system of computer programs designed to perform geocoding to a variety of records. However, we were unable to use this system of programs due to a host of difficulties specific to our needs.

In the following paragraphs, we present a description of a system of three computer programs that can be viewed as an alternative to the ADMATCH system. Like ADMATCH, our system of programs is designed to match records from two distinct files: a master list of addresses with the appropriate geographical codes and the individual records to which these codes are to be assigned. This system of programs is used to transfer a tract and block code from a master address list containing these codes to address records not previously containing them. Generally speaking, this master list of addresses is any reference file containing both addresses and geographic codes specific to those addresses. The computer programs are written in ANSI-COBOL. The interrelationships among these programs are shown in Figure 1.

Program 1: Select Census Record-Core Required$37 \mathrm{~KB}$. The Select Census Record program reads the master address file (in this case, the census GBF-DIME file, which is a specific reference file) and selects all desired records as defined by zip code. ${ }^{1}$ The census file has a range of house numbers for the left side of the street and a range of numbers for the right side, with the left side being odd and the right side even. Therefore, two records are written for each street segment selected. The odd numbers have an assigned type code of 1 , and even numbers have an assigned type code of 2 . The output is condensed, containing only those fields

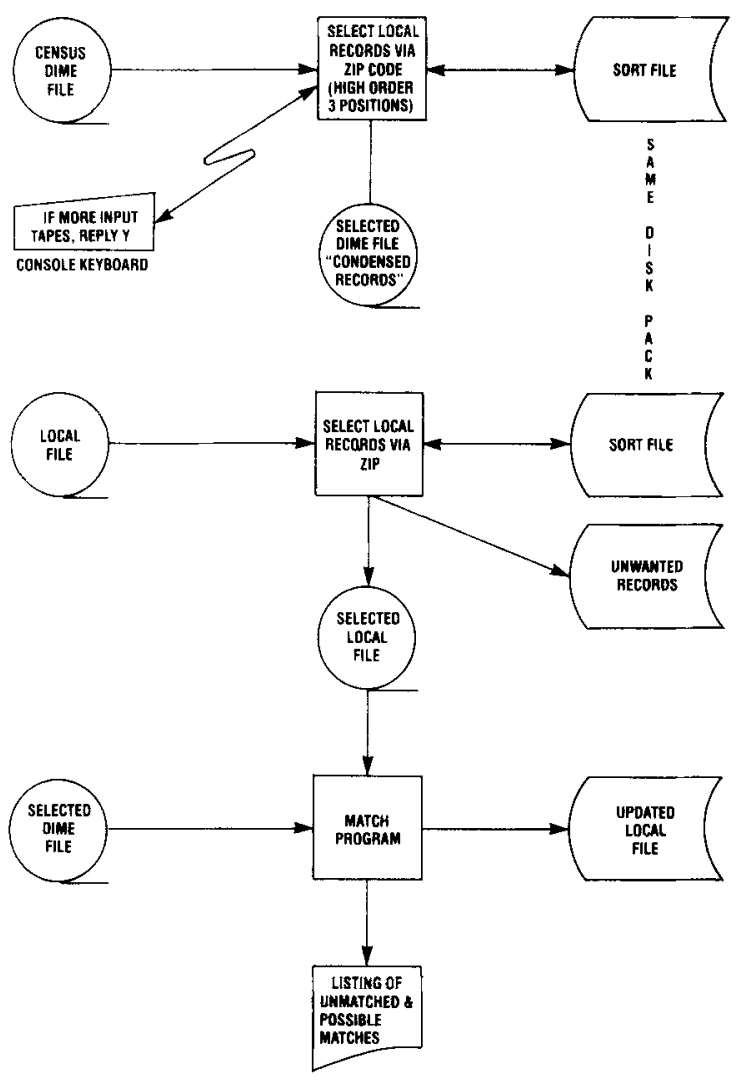

Figure 1. Flowchart for match system.

needed for matching, plus tract and block numbers. The output also contains the address as it appears in the census GBF-DIME file and the address as it appears in the local address file (addresses one wishes to match), thus providing two separate formats by which to match records.

The output file is sorted into sequence according to the following characteristics, listed in order of importance (major to minor): (1) record type (1 or 2), (2) street name (exact name, disregarding N., S., E., St., Rd., $\mathrm{Cv}$., etc.), (3) lowest house number, and (4) zip code. Zip code is used for sorting, in order to separate streets in different areas that have the same name and series of house numbers. The output file from the Select Census Record program is input to Program 3, the Match Selected Records program.

Program 2: Select Local Records-Core Required$53 \mathrm{~KB}$. The Select Local Records program reads the local file (in this case, real estate records) and selects the desired records as defined by zip code. It also attempts to format the address as it appears in the census file and assigns record type codes ( 1 for odd and 2 for even).

The output file is written in sorted sequence by record type, street name (reformatted exact name), 
street number, and zip code. This file is also input to Program 3, the Match Selected Records program. If requested, a file of undesired records can be output to disk for future use.

Program 3: Match Selected Records-Core Required$29 \mathrm{~KB}$. The Match Selected Records program matches the two output files created by Programs 1 and 2 using a point evaluation system. The point system corresponds to the sort sequence, giving a higher value to the major sort field and a lower value to the minor sort field: (1) match on record type, value $=8$;(2) match on street name, value $=4 ;$ (3) match on street number, value $=2$; (4) match on zip code, value $=1$. These point values were purposely selected, as the sum of any combination of fields (two or three fields) will be a unique sum. A record is considered to be exactly matched when the total point value equals $6,7,14$, or 15 . A 13 is defined as a possible match, and all other combinations (sums) are defined as unmatched. All local records are written out, and those that are matches or possible matches are written with the census tract and block numbers.

The Match Selected Records program always holds the previous census record in a "save" area as it advances the census file. If it does not find an exact match on the current record, it checks to see if the previous record provides a better match. If neither is satisfactory, it advances the census file and checks again, as long as the match fields in the census file are not greater than those in the local file. A printed list of those records having no match is also provided.

\section{Relative Advantages}

The major advantage of this system of programs is its realtive simplicity. Anyone with a minimum knowledge of COBOL can modify the programs to process his or her files. Furthermore, as far as modification is concerned, the Select Local Records program must be changed only with respect to record length, block length, location, and length of fields in order to meet the individual requirements of one's own data files. All that is required in the Select Census Records program is a change in range of the zip codes that one is selecting. If one wishes to process the complete file, the "zip code compare" statement may be removed. The only modification to the Match Selected Records program is to the "select county" file, which one must define exactly as the output file from the Select Local Records program. No additional passes of unmatched records are required, as our system of programs selects the best possible match on the initial pass.

\section{Verification and Accuracy}

The system of programs reviewed here has been verified by matching a local real estate file containing approximately 153,000 records to the U.S. Census Bureau's geographic base file (GBF-DIME) of addresses. A match rate of approximately $95 \%$ was obtained, and this rate is comparable to that of the U.S. Census Bureau's (1970) ADMATCH programs.

The programs were tested on an IBM Model 360/30 computer having $64 \mathrm{~KB}$ of storage (of which $10 \mathrm{~KB}$ is used by the systems supervisor) with three 2314 disk drives and two 2400 tape drives. These programs have since been run on a Univac 1100 , with the only modification required being the appropriate changes in JCL. Of course, core requirements for the present programs could be reduced by (1) using utility rather than COBOL sorts, (2) reducing the blocking factor of the files, and/ or (3) reserving no alternative areas for input/output. However, the tradeoff would be slower processing. On the other hand, it should be noted that larger core storage availability could significantly speed up processing.

\section{Availability}

A printed listing of these programs is available at no cost. Contact either Rebecca F. Guy or Louis G. Pol, Department of Sociology, Memphis State University, Memphis, Tennessee 38152.

\section{REFERENCE}

U.S. Bureau of the Census. aDMATCH users manual. Washington, D.C: U.S. Government Printing Office, 1970.

\section{NOTE}

1. The GBF-DIME file is the U.S. Bureau of the Census Geographic Based File for Direct Independent Map Encoding. This file contains street address ranges, as well as a host of geographic identifying codes specific to the addresses within those ranges.

(Accepted for publication May 29, 1982.) 